\title{
Effect of antihypertensive therapy on hospitalization and mortality among uncomplicated and high risk hypertensive patients
}

\author{
Luca Degli Esposti ${ }^{{ }^{*}}$, Stefania Saragoni ${ }^{1}$, Chiara Veronesi ${ }^{1}$, Carlo Cerra $^{2}$, Paolo Batacchi ${ }^{3}$, \\ Claudia Pagliaro ${ }^{4}$, Alessandra Sturani ${ }^{5}$, Ezio Degli Esposti ${ }^{1}$ \\ ${ }^{1}$ CliCon S.r.l. Health, Economics and Outcomes Research, Ravenna, Italy; ${ }^{*}$ Corresponding Author: luca.degliesposti@,clicon.it \\ ${ }^{2}$ Information System, Local Health Unit of Pavia, Pavia, Italy \\ ${ }^{3}$ Pharmaceutical Policy Department, Local Health Unit of Florence, Florence, Italy \\ ${ }^{4}$ Department Internal Management Control, Local Health Unit of Caserta, Caserta, Italy \\ ${ }^{5}$ Department of Nephrology, S. M. delle Croci Hospital, Ravenna, Italy
}

Received 21 February 2013; revised 26 March 2013; accepted 4 April 2013

Copyright (C) 2013 Luca Degli Esposti et al. This is an open access article distributed under the Creative Commons Attribution License, which permits unrestricted use, distribution, and reproduction in any medium, provided the original work is properly cited.

\section{ABSTRACT}

Objective: Evaluate the impact of antihypertensive drugs (AHD) on stroke and acute myocardial infarction (AMI) and death for 3 cohorts of patients: diabetics, high risk, and hypertensive. Methods: This was a retrospective cohort observational study based on administrative database of 7 Italian Local Health Units. Newly treated patients with AHD were enrolled from January 1, 2004 to June 30, 2007. Drug utilization and incidence of death, stroke and AMI were measured until December 12, 2007. Results: The study enrolled 125,770 patients aged $\geq 18$-year-old: 8516 diabetic, 8549 high risk, and 108,705 hypertensive. Diabetic and high risk patients were more frequently male $(57.2 \%, 64.9 \%$ VS $46.6 \%)$ and older $(66.4 \pm 11.6,67.5 \pm 13.2$ VS $61.6 \pm 13.9)$ than hypertensive patients and were more treated with concomitant therapy. Drugs acting on the renin-angiotensin system were the more frequent choice in the three cohorts but with a different prevalence $(63.9 \%, 52.9 \%, 35.9 \%$ in diabetic, hypertensive and high risk patients respectively). Adherent patients to AHD (PDC > $80 \%$ ) were $44.0 \%$ among diabetic patients, $48.4 \%$ among high risk, $35.2 \%$ among hypertensive. A Cox regression model showed that the risk of the combined outcome was determined by increasing age, male gender, presence of concomitant therapy, low adherence to AHD and first choice of AHD. Conclusions: An integrated analysis of prescription and hospital discharge database has the great advantage that it uses routinely available data, it can be done quickly and by few staff, and it is less expensive than planned large scale survey.

Keywords: Component; Formatting; Style; Styling; Insert

\section{INTRODUCTION}

Elevated blood pressure (BP) is still the biggest single contributor to global mortality [1], and extensive randomized trial data are consistent in showing that BP reduction substantially reduces cardiovascular $(\mathrm{CV})$ morbidity and mortality [2,3], and demonstrate clear benefit across many subgroups of hypertensive patients [4]. These data justifies to start the administration of BP lowering drugs in uncomplicated hypertensive patients as well as in hypertensive patients with associated $\mathrm{CV}$ disease or diabetes, as recommended by recent guidelines $[5,6]$. In clinical practice, under-treatment of high BP [7] and poor adherence to antihypertensive drugs (AHD) [8] cause suboptimal BP control and, ultimately, preventable CV morbidity and mortality. The true dimension of the problem, however, is not well known: accurate estimates of prevalence, awareness, treatment, and control of high BP required detailed, complex, and expensive population survey, and it is usually necessary to restrict such survey to a limited group of subjects and selected periods [9]. An integrated analysis of prescription, hospital discharges and mortality database could offer the opportunity to carry out valuable research and clinical audit and provide accurate estimates of prevalence, drugs' man- 
agement and outcome of care.

The main purpose of our survey was to identify uncomplicated hypertensive patients as well as hypertensive patients with associated CV disease or diabetes using data from administrative database, and to evaluate the relationship between the antihypertensive treatment and the incidence of stroke, acute myocardial infarction (AMI) and death in the three cohorts of patients.

\section{PATIENTS AND METHODS}

\subsection{Data Source}

The study was based on administrative databases maintained by 7 Local Health Units (LHU) in Italy, belonging to Lombardy, Veneto, Tuscany, Campania, Sicily, Sardinia regions, with a population of approximately 3,000,000 inhabitants. The LHU Ethics Committees approved the study. In the Medications Prescription Database, the LHU routinely measures the volume of expenditure generated by the dispensing of drugs to the enrollers. The data available in each prescription claim include the patient's national health number, the prescribing physician's number, the anatomical-therapeutic-chemical (ATC) code of the drug delivered, the number of packs, the number of units per pack, the dosage, the unit cost per pack, and the prescription date. Using the numeric code released to each citizen by the LHU as a unique identifier, this database was linked with the Beneficiaries' Database, listing some patients' demographic characteristics i.e. date of birth, sex, place of residence, physician license number, start and end of registration dates, and the Hospital Discharge Database, which includes all hospitalization data with the discharge diagnosis codes classified according to the International Classification of Diseases, Ninth Revision (ICD9CM), and the Mortality Database, where death data are recorded. It was not possible to retrieve the cause of death from death certificates. Universal health-care coverage in Italy allows completeness and comprehensiveness of the information contained in these databases, which have been used in previous epidemiological studies [10]. The Italian Ministry of Health reported that archives are $100 \%$ complete and $95 \%$ accurate [11]. In order to guarantee patient privacy, each subject was assigned an anonymous univocal alphanumeric code.

\subsection{Cohort Definition}

This was a retrospective cohort study, which included only new AHT users, aged over 18 years old, with a complete history of prescriptions and clinical outcome data over the study period. Subjects were enrolled if they had at least one prescription of AHT (diuretics [D, ATC code C03], excluding loop diuretics [ATC code $\mathrm{C} 03 \mathrm{C}$ ], betablockers [BB, ATC code C07], calcium-channel blockers
[CCB, ATC code C08], angiotensin-converting enzyme inhibitors [ACE I, ATC code C09A/B], angiotensin-receptor blockers [ARB, ATC code C09C/D], or other antihypertensive drugs [ATC code C02]) dispensed between January 1, 2004 and June 30, 2007 (enrolment period). The date of the first AHT claim was defined as the enrolment date. We excluded records of subjects who died, moved to other LHU or were hospitalized with a diagnosis of stroke or AMI in the 6 months after the enrolment date, in order to ensure a minimum time interval for adherence assessment: in fact, the use of short-term time intervals to assess adherence has been shown not to reflect accurately long-term behaviours $[12,13]$. Subjects were defined new users if they had not been prescribed any AHD in the year before the enrolment date. Subjects were defined as diabetic if they had received at least two prescriptions of hypoglycaemic drugs (ATC codes A10) in the year before the enrolment date and as high risk if they were hospitalized for hypertension (ICD9CM codes 401 - 405), ischemic heart disease (ICD9CM codes 410 414), heart failure (ICD9CM code 428), cerebrovascular disorders (ICD9CM codes 430 - 438), or vascular diseases (ICD9CM codes 440 - 442), and those prescribed with nitrates (ATC code C01DA) in the year before the enrolment date [14]. The remaining subjects were defined as hypertensive. The prescriptions of hypolipidemic drugs [ATC code C10], cardiac drugs [ATC code C01], antiplatelet drugs [ATC code B01AC] and loop diuretic drugs in the 12 months before the enrolment date were evaluated in the three cohorts of patients.

\subsection{Adherence to AHT}

Adherence to AHD was estimated as the percentage of days a subject had tablets available (proportion of days covered, PDC), from the first delivery of AHD until either death, moving to another LHU, hospitalization for AMI or stroke, or June 30, 2007, whichever occurred earlier. The interval was separated into treatment episodes of continuous AHD use based on the method of Catalan and LeLorier [15]. A treatment episode was measured as the time-span between the starting dates of the first AHT dispensing until the last day of the final AHD supply. The latter date included allowance for a possible gap after the final dispensing within the specific episode. Prescriptions filled near the end of the interval contributed days till that date. Prescriptions containing more than 1 drug contributed 1) the sum of the days' supply of all drugs, in case of drugs from the same AHD drug class, because a possible stockpiling of medication was considered; 2) the lower days' supply drug value, in case of drugs from different AHD drug classes, identifying them as a combined therapy. The PDC corresponded to the total of number of days' supply of medication dispensed within each episode, divided by the total length 
of the interval and multiplied by 100. Subjects were grouped into 4 adherence categories as follows: PDC $\leq$ $40 \%$, ranging from $41 \%$ to $60 \%$, ranging from $61 \%$ to $80 \%$, and PDC $>80 \%$. When PDC was $\leq 20 \%$, indication for AHD was considered uncertain or questionable and, therefore, the corresponding subjects were excluded [16].

\subsection{Outcomes}

The primary study outcome was all-cause mortality or the first hospitalization for fatal or nonfatal AMI (ICD9CM codes 410.x) or stroke (ICD9CM codes 430 through 438.x). Patients were followed-up from the date of enrolment until the earliest of the following end dates: hospital discharge for AMI or stroke; death from any cause; study closure (December 31, 2007); or cancellation from the enrollees' list in the LHUs.

\subsection{Statistical Analysis}

Data were summarized as mean \pm standard deviation (SD) for continuous variable and as numbers (percentages) of subjects for categorical variables. Pearson's chisquare and one-way ANOVA tests were used to evaluate differences in baseline characteristics across the three cohorts of patients. Multivariable Cox proportional hazard regression model was used to estimate hazard ratios (HR) and 95\% confidence intervals (CI) of death and CV events, as combined outcomes, as a function of the three cohorts of patients. To adjust for potential confounders, in the multivariable model we included age, gender, and presence of specific treatments in the year prior to the enrolment date, taken as proxies for the diagnoses of dyslipidemia (ATC code C10), heart disease (ATC code C01), and atherosclerotic disease (ATC code B01AC). Visual inspection of the survival curves confirmed that the assumption of proportional hazards was not violated. Two-tailed p-values $<0.05$ were considered statistically significant. All analyses were conducted using SPSS for Windows, version 18.0.

\section{RESULTS}

Out of a population of 1,867,511 subjects aged over 18 years old, 253,972 subjects $(13.6 \%)$ had their first anti-hypertensive prescription filled from January 1, 2004 through June 30, 2007, and did not moved from the LHUs along the follow-up period.

Of these, 128,202 study participants $(50.5 \%$ of the enrolled subjects) were excluded: 7308 (2.9\%) because they had a study outcome during the 6 months after the enrolment date, and 120,894 (47.6\%) because they had a PDC $<20 \%$ due to occasional or intermittent prescriptions of AHD or to a therapy lasting less than 6 months. A total of 125,770 subjects $(49.5 \%$ of the enrolled subjects) were included in the study: $8516(6.8 \%$ of the included cohort), mean age $66.4 \pm 11.6$ years, were defined as diabetic patients, $8549(6.8 \%)$, mean age $67.5 \pm 13.2$ years, as high risk patients, and 108,705 (86.4\%), mean age $61.6 \pm 13.9$ years, as hypertensive patients. Some characteristics of the patients and the use of concomitant drug treatment are shown in Table 1. Diabetic and high risk patients were significantly $(p<0.001)$ more frequently male and older than hypertensive patients and were significantly $(\mathrm{p}<0.001)$ more treated with concomitant therapy. In the diabetic cohort, 316 patients (3.7\%) received prescriptions of nitrates and 854 (10\%) had had previous hospital discharges for $\mathrm{CV}$ diseases.

The starting antihypertensive therapy was significantly different $(p<0.001)$ among the three cohorts of patients (Table 2). Drugs acting on the renin-angiotensin system (ACE I and ARB) were the more frequent choice in the three cohorts but their prevalence resulted significantly different among the groups $(63.9 \%, 52.9 \%, 35.9 \%$ in diabetic, hypertensive and high risk patients respectively). In each cohort ACEs I were significantly $(p<0.001)$ more prescribed than ARBs. In diabetic patients the second choice was Ds (12.2\%), in high risk patients was the association of two antihypertensive drugs (27.9\%), and in hypertensive patients was BBs (16.4\%).

Table 1. Gender and concomitant therapy in enrolled patients.

\begin{tabular}{|c|c|c|c|c|c|c|}
\hline & \multicolumn{6}{|c|}{ Patients } \\
\hline & \multicolumn{2}{|c|}{ Diabetic } & \multicolumn{2}{|c|}{ High risk } & \multicolumn{2}{|c|}{ Hypertensive } \\
\hline & $\mathrm{n}$ & $\%$ & $\mathrm{n}$ & $\%$ & $\mathrm{n}$ & $\%$ \\
\hline Male gender & 4875 & 57.2 & 5545 & 64.9 & 50,642 & 46.6 \\
\hline Hypolipemic therapy & 1445 & 17.0 & 1008 & 11.8 & 5472 & 5.0 \\
\hline Cardiac therapy & 512 & 6.0 & 1608 & 18.8 & 2652 & 2.4 \\
\hline Antiplatelet therapy & 1690 & 19.8 & 1892 & 22.1 & 6180 & 5.7 \\
\hline Nitrates therapy & 316 & 3.7 & 2087 & 24.4 & 0 & 0.0 \\
\hline Loop diuretic therapy & 1460 & 17.1 & 2340 & 27.4 & 11,117 & 10.2 \\
\hline Previous CV hospitalizations & 854 & 10.0 & 6794 & 79.5 & 0 & 0.0 \\
\hline
\end{tabular}


Table 2. Starting antihypertensive therapy in enrolled patients.

\begin{tabular}{ccccccc}
\hline & \multicolumn{3}{c}{ Patients } \\
\cline { 2 - 7 } Starting drug & \multicolumn{2}{c}{ Diabetic } & \multicolumn{3}{c}{ High risk } & \multicolumn{3}{c}{ Hypertensive } \\
\cline { 2 - 7 } & $\mathrm{n}$ & $\%$ & $\mathrm{n}$ & $\%$ & $\mathrm{n}$ & $\%$ \\
\hline D & 1036 & 12.2 & 1090 & 12.8 & 15,074 & 13.9 \\
BB & 652 & 7.7 & 1362 & 15.9 & 17,809 & 16.4 \\
ACE I & 3768 & 44.2 & 2577 & 30.1 & 39,040 & 35.9 \\
ARB & 1677 & 19.7 & 500 & 5.8 & 18,514 & 17.0 \\
CCB & 600 & 7.0 & 632 & 7.4 & 9945 & 9.1 \\
Associations & 783 & 9.2 & 2388 & 27.9 & 8323 & 7.7 \\
Total & 8516 & 100.0 & 8549 & 100.0 & 108,705 & 100.0 \\
\hline
\end{tabular}

The adherence to AHD in the three cohorts of patients was reported in Table 3. The percentage of subjects adherent to the AHD treatment increased significantly along the levels of adherence in high risk and diabetic cohorts reaching the best level (PDC $>80 \%$ ) in $48.4 \%$ and $44.0 \%$ of patients respectively. In the hypertensive cohort the prevalence $(35.2 \%)$ of subjects with the highest level of adherence was significantly lower $(p<0.001)$ than in the diabetic and high risk cohorts, with no significant difference in the prevalence of other three levels of adherence.

A total of 8760 outcomes were observed during the follow-up, including 5900 deaths, 2112 strokes, and 748 AMI. Incidence of the combined outcome as well as of each single outcome was significantly higher in high risk and diabetic patients compared with hypertensive patients (Table 4 and Figure 1). The multiregression analysis (Table 5) showed that the risk of the combined outcome is determined by the same factors in the three cohorts of subjects even if at different extents: it increased by ageing, male gender, presence of concomitant therapy excepted lipid lowering drugs, decreased adherence to AHD and first choice of AHD.

\section{DISCUSSION}

A positive benefit of observational studies is that they offer the opportunity to carry out clinical audit and evaluative research, inform the planning and management of services, and provide clinicians with accurate estimates of the outcome of care. They represent a complementary approach rather than an alternative to clinical trials and can contribute to evaluate the health care in everyday practice and hence how to improve organization of services and individual pharmacological treatment [17].

Information provided by prescription database enables us to perform good quality observational studies. Our survey has shown that prescription of AHD analysis can be a useful way of establishing disease prevalence of hypertensive patients. Moreover, integrating the analysis with prescription of hypoglycaemic and/or nitrate drugs and hospital discharge, it was possible to identify hypertensive patients at elevated $\mathrm{CV}$ risk as diabetic patients and subject who had suffered from CV diseases or events. This procedure was possible because the use of AHD, hypoglycaemic and nitrates drugs was quite specific to hypertension, diabetes and ischemic disease respectively, thus the contemporary analysis of the three classes of drugs, together with hospital discharges for CV reasons, improves the sensitivity in detecting patients cohorts.

Existing publication focus generally on single cohort of patients $[8,16,18-21]$. We have chosen to compare different cohorts of hypertensive patients managed in the same geographical, clinical and organizational setting in order to have a comprehensive evaluation of the real world. As expected, the incidence of the composite outcome was significantly different in three cohorts: $12.3 \%$ in diabetic, $16.8 \%$ in high risk, and $5.8 \%$ in hypertensive patients, but the absolute number of subjects affected was 5 - 6 times higher in hypertensive $(6280$ subjects) than in diabetic (1045 subjects) and high risk (1435 subjects) patients.

We have enrolled in the study only patients who were regularly taking AHD, and this methodology may have contribute to an underestimation of the prevalence, but for disease management as well as for planning allocation of resources, it is the number of patients who present to a doctor and need drug treatments and/or hospital care that matters $[22,23]$. Moreover, the factor determining the composite outcome measured in the present study was similar in the three cohorts of patients: aging, concomitant medications (as proxy of comorbidities), adherence to AHD, but the weight of the each factor was quite different. In diabetic patients only an adherence > $80 \%$ contribute to reduce the risk of outcomes, while in high risk patients increasing adherence progressively decrease risk of death or $\mathrm{CV}$ events.

In hypertensive patients an age over 65 years and male gender seem to contribute significantly to the incidence of combined outcome.

The identification of the negative or positive influence 
Table 3. Adherence to antihypertensive therapy.

\begin{tabular}{ccccccc}
\hline \multicolumn{7}{c}{ Patients } \\
\hline & \multicolumn{2}{c}{ Diabetic } & \multicolumn{2}{c}{ High risk } & \multicolumn{2}{c}{ Hypertensive } \\
\hline Adherence level & $\mathrm{n}$ & $\%$ & $\mathrm{n}$ & $\%$ & $\mathrm{n}$ & $\%$ \\
\hline$\leq 40 \%$ & 1498 & 17.6 & 1337 & 15.6 & 24,905 & 22.9 \\
$41 \%-60 \%$ & 1551 & 18.2 & 1415 & 16.6 & 23,063 & 21.2 \\
$61 \%-80 \%$ & 1716 & 20.2 & 1661 & 19.4 & 22,422 & 20.6 \\
$>80 \%$ & 3715 & 44.0 & 4136 & 48.4 & 38,315 & 35.2 \\
Total & 8516 & 100.0 & 8549 & 100.0 & 108,705 & 100.0 \\
\hline
\end{tabular}

Table 4. Incidence of outcomes in the three cohorts of subjects.

\begin{tabular}{ccccccc}
\hline \multirow{2}{*}{ Outcome } & \multicolumn{9}{c}{ Patients } \\
\cline { 2 - 7 } & \multicolumn{2}{c}{ Diabetic } & \multicolumn{2}{c}{ High risk } & \multicolumn{2}{c}{ Hypertensive } \\
\cline { 2 - 7 } & $\mathrm{n}$ & $\%^{1}$ & $\mathrm{n}$ & $\%^{2}$ & $\mathrm{n}$ & $\%^{3}$ \\
\hline Death & 658 & 7.7 & 941 & 11.0 & 4301 & 4.0 \\
Stroke & 263 & 3.1 & 391 & 4.6 & 1458 & 1.3 \\
AMI & 124 & 1.5 & 103 & 1.2 & 521 & 0.5 \\
Total & 1045 & 12.3 & 1435 & 16.8 & 6280 & 5.8 \\
\hline
\end{tabular}

${ }^{1}$ Calculated in relation to 8516 diabetic patients; ${ }^{2}$ Calculated in relation to 8549 high risk patients; ${ }^{3}$ Calculated in relation to 108,705 hypertensive patients.

Combined event + Death

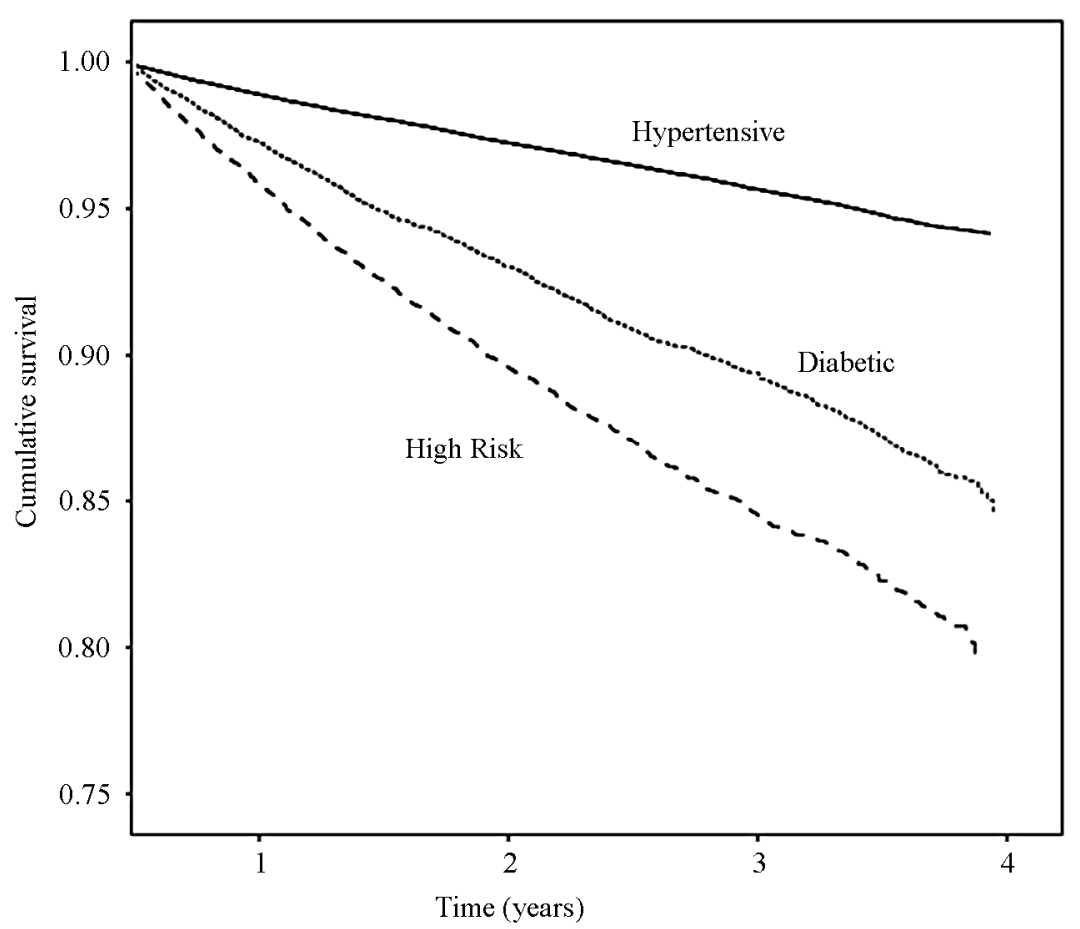

Figure 1. Combined outcome of all-cause death, stroke and AMI curves by disease-specific cohorts.

connected to correctable and non correctable factors in the three cohorts would allow the improvement of the care of patients. It is relevant that in the three cohorts the presence of a treatment with lipid lowering drugs reduces the risk of event: unfortunately, in the present survey the percentage of patients receiving prescriptions of these drugs is very low ranging from $17.0 \%$ in diabetic patients to $5.0 \%$ in hypertensive patients. Regarding the 
Table 5. Multivariate analysis of the association of patient characteristics, concomitant medications, adherence to antihypertensive therapy and starting antihypertensive drug with first study outcome.

\begin{tabular}{|c|c|c|c|c|c|c|}
\hline \multirow{3}{*}{ variable } & \multicolumn{6}{|c|}{ Patients } \\
\hline & \multicolumn{2}{|l|}{ Diabetic } & \multicolumn{2}{|l|}{ High risk } & \multicolumn{2}{|l|}{ Hypertensive } \\
\hline & Hazard ratio $(95 \% \mathrm{CI})$ & $\mathrm{p}<$ & Hazard ratio $(95 \% \mathrm{CI})$ & $\mathrm{p}<$ & Hazard ratio $(95 \% \mathrm{CI})$ & $\mathrm{p}<$ \\
\hline Age, years & & 0.001 & & 0.001 & & 0.001 \\
\hline$<45^{1}$ & 1.00 & & 1.00 & & 1.00 & \\
\hline $45-64$ & $1.21(0.71-2.04)$ & NS & $1.15(0.71-1.87)$ & NS & $1.98(1.68-2.33)$ & 0.001 \\
\hline$\geq 65$ & $2.97(1.77-4.98)$ & 0.001 & $3.18(1.99-5.10)$ & 0.001 & $6.66(5.69-7.80)$ & 0.001 \\
\hline \multicolumn{7}{|l|}{ Gender } \\
\hline Female $^{1}$ & 1.00 & & 1.00 & & 1.00 & \\
\hline Male & $1.23(1.08-1.39)$ & 0.01 & $1.06(0.95-1.18)$ & NS & $1.55(1.48-1.63)$ & 0.001 \\
\hline \multicolumn{7}{|l|}{ Medications $^{2}$} \\
\hline Hypolipemic & $0.68(0.56-0.83)$ & 0.001 & $0.50(0.40-0.62)$ & 0.001 & $0.60(0.53-0.69)$ & 0.001 \\
\hline Cardiac & $1.26(1.04-1.53)$ & 0.01 & $1.35(1.18-1.56)$ & 0.001 & $1.35(1.22-1.49)$ & 0.001 \\
\hline Antiplatelet & $1.34(1.17-1.54)$ & 0.001 & $1.13(0.99-1.27)$ & NS & $1.44(1.33-1.55)$ & 0.001 \\
\hline Loop diuretic & $1.83(1.58-2.13)$ & 0.001 & $1.27(1.13-1.44)$ & 0.001 & $2.67(2.51-2.83)$ & 0.001 \\
\hline CV events & $1.33(1.11-1.58)$ & 0.01 & $1.28(1.11-1.47)$ & 0.01 & & \\
\hline Adherence, $\%$ & & 0.01 & & 0.001 & & 0.001 \\
\hline$\leq 40^{1}$ & 1.00 & & 1.00 & & 1.00 & \\
\hline $41-60$ & $0.88(0.73-1.06)$ & NS & $0.81(0.69-0.94)$ & 0.01 & $0.84(0.78-0.90)$ & 0.001 \\
\hline $61-80$ & $0.85(0.69-1.02)$ & NS & $0.73(0.62-0.85)$ & 0.001 & $0.81(0.75-0.87)$ & 0.001 \\
\hline$>80$ & $0.76(0.64-0.91)$ & 0.01 & $0.55(0.47-0.63)$ & 0.001 & $0.73(0.68-0.78)$ & 0.001 \\
\hline Starting drug & & 0.001 & & 0.001 & & 0.001 \\
\hline $\mathrm{ARB}^{1}$ & 1.00 & & 1.00 & & 1.00 & \\
\hline $\mathrm{D}$ & $2.13(1.69-2.69)$ & 0.001 & $1.78(1.38-2.30)$ & 0.001 & $1.88(1.71-2.06)$ & 0.001 \\
\hline BB & $1.28(0.95-1.73)$ & NS & $0.68(0.50-0.91)$ & 0.05 & $0.95(0.85-1.07)$ & NS \\
\hline $\mathrm{CCB}$ & $1.58(1.20-2.08)$ & 0.01 & $1.39(1.04-1.85)$ & 0.05 & $1.40(1.25-1.56)$ & 0.001 \\
\hline ACE I & $1.16(0.94-1.42)$ & NS & $1.28(1.00-1.63)$ & 0.05 & $1.12(1.02-1.22)$ & 0.05 \\
\hline Association & $1.59(1.23-2.06)$ & 0.001 & $1.05(0.80-1.35)$ & NS & $1.31(1.18-1.47)$ & 0.001 \\
\hline
\end{tabular}

${ }^{1}$ Reference value; ${ }^{2}$ Absence of medication as reference value; $\mathrm{ARB}=$ angiotensin $\mathrm{II}$ receptor blockers; $\mathrm{D}=$ diuretics; $\mathrm{BB}=$ beta blockers; $\mathrm{CCB}=$ calcium channels blockers; ACE I = angiotensin converting enzyme inhibitors.

starting AHD therapy, we have chosen as reference ARB because they have an antihypertensive effect similar to other classes of AHD but less side effects [24] and more adherence to treatment $[25,26]$. The use of diuretics as starting drug increases the risk of outcomes in the three cohorts. In diabetic patients there were no significant differences among ARB, ACE I, and BB; in high risk patients there were no significant differences among $\mathrm{ARB}$ and association of two or more drugs, BB had a protective effect; in hypertensive patients there were no significant differences among ARB and BB.

In conclusion, an integrated analysis of prescription and hospital discharge database has the great advantage that it uses routinely available data, it can de done quickly and by few staff, and it is less expensive than planned large scale survey.

\section{REFERENCES}

[1] Ezzati, M., Lopez, A.D., Rodgers, A., et al. (2002) Selected major risk factors and global and regional burden of disease. Lancet, 360, 1347-1360. doi:10.1016/S0140-6736(02)11403-6

[2] Blood Pressure Lowering Treatment Trialists Collaboration (2003) Effects of different blood-pressure-lowering regimens on major cardiovascular events: Results of prospectively-designed overviews of randomized trials. Lancet, 362, 1527-1535.

[3] Law, M.R., Morris, J.K. and Wald, N.J. (2009) Use of blood pressure lowering drugs in the prevention of cardiovascular disease: Meta-analysis of 147 randomised trials in the context of expectations from prospective epidemiological studies. British Medical Journal, 338.

[4] Neal, B., MacMahon, S. and Chapman, N. (2000) Effects 
of ACE inhibitors, calcium antagonists, and other bloodpressure-lowering drugs: Results of prospectively designed overviews of randomised trials. Lancet, 356, 1955-1964. doi:10.1016/S0140-6736(00)03307-9

[5] The Task Force for the Management of Arterial Hypertension of the European Society of Hypertension (ESH) and of the European Society of Cardiology (ESC) (2007) 2007 Guidelines for the management of arterial hypertension. European Heart Journal, 28, 1462-1536.

[6] Campbell, N.R.C., Khan, N.A., Hill, M.D., et al. (2009) On the behalf of the canadian hypertension education program. 2009 Canadian hypertension education program recommendations: The scientific summary-An annual update. Canadian Journal of Cardiology, 25, 271-277. doi:10.1016/S0828-282X(09)70490-8

[7] Degli Esposti, L. and Valpiani, G. (2004) Pharmaco-economic burden of undertreating hypertension. Pharmacoeconomics, 22, 907-928. doi:10.2165/00019053-200422140-00002

[8] Degli Esposti, L., Saragoni, S., Benemei, S., et al. (2011) Adherence to antihypertensive medications and health outcomes among newly treated hypertensive patients. Journal of ClinicoEconomics and Outcomes Research, 3, 47-54. doi:10.2147/CEOR.S15619

[9] Egan, B.M., Zhao, Y. and Axon, R.N. (2010) US trends in prevalence, awareness, treatment, and control of hypertension, 1988-2008. JAMA, 303, 2043-2050. doi:10.1001/jama.2010.650

[10] Di Bari, M., Balzi, D., Roberts, A.T., et al. (2010) Prognostic stratification of folder persons based on simple administrative data: Development and validation of the "Silver Code", to be used in emergency department triage. Journals of Gerontology Series A: Biological Sciences and Medical Sciences, 65, 159-164. doi:10.1093/gerona/glp043

[11] Ministero del Lavoro, della Salute e delle Politiche Sociali (2005) Rapporto annuale sulle attivita' di ricovero ospedaliero.

http://www.ministerosalute.it/programmazione/sdo/sezDo cumenti.jsp?id $=148 \&$ label $=$ osp

[12] Rasmussen, J.N., Chong, A. and Alter, D.A. (2007) Relationship between adherence to evidence-based pharmacotherapy and long-term mortality after acute myocardial infarction. JAMA, 297, 177-186. doi:10.1001/jama.297.2.177

[13] Suissa, S. (2007) Immortal time bias in observational studies of drug effects. Pharmacoepidemiology Drug Safety, 16, 241-249. doi:10.1002/pds. 1357

[14] Clarke, K.W., Gray, D. and Hampton, J.R. (1994) Implication of prescriptions for nitrates: 7 year follow up of patients treated for angina in general practice. British Heart Journal, 71, 38-40. doi:10.1136/hrt.71.1.38

[15] Catalan, V.S. and LeLorier, J. (2000) Predictors of longterm persistence on stations in a subsidized clinical population. Value Health, 3, 417-426. doi:10.1046/j.1524-4733.2000.36006.x

[16] Kettani, F.Z., Dragomir, A., Cote, R., et al. (2009) Impact of better adherence to antihypertensive antihypertensive agents on cerebrovascular disease for primary prevention. Stroke, 40, 213-220.

doi:10.1161/STROKEAHA.108.522193

[17] Black, N. (1996) Why we need observational studies to evaluate the effectiveness of health care. British Medical Journal, 312, 1215-1218. doi:10.1136/bmj.312.7040.1215

[18] Ho, P.M., Rumsfeld, J.S., Masoudi, F.A., et al. (2006) Effect of medication nonadherence on hospitalization and mortality among patients with diabetes mellitus. Archives of Internal Medicine, 166, 1836-1841. doi:10.1001/archinte.166.17.1836

[19] Rasmussen, J.N., Chong, A. and Alter, D.A. (2007) Relationship between adherence to evidence-based pharmacotherapy and long-term mortality after acute myocardial infarction. JAMA, 297, 177-186. doi:10.1001/jama.297.2.177

[20] Gislason, G.H., Rasmessen, J.N., Abildstrom, S.Z., et al. (2007) Persistent use of evidence-based pharmacotherapy in heart failure is associated with improved outcomes. Circulation, 116, 737-744. doi:10.1161/CIRCULATIONAHA.106.669101

[21] Mazzaglia, G., Ambrosioni, E., Alacqua, M., et al. (2009) Adherence to antihypertensive medications and cardiovascular morbidity among newly diagnosed hypertensive patients. Circulation, 120, 1598-1605. doi:10.1161/CIRCULATIONAHA.108.830299

[22] Sokol, M.C., McGuigan, K.A., Verbrugge, R.R., et al. (2005) Impact of medication adherence on hospitalization risk and healthcare cost. Medical Care, 43, 521-530. doi:10.1097/01.mlr.0000163641.86870.af

[23] Leal, J., Luengo-Fernandez, Gray, A., et al. (2006) Economic burden of cardiovascular diseases in the enlarged European Union. European Heart Journal, 27, 16101619. doi:10.1093/eurheartj/ehi733

[24] Law, M.R., Wald, N.J., Morris, J.K. and Jordan, R.E. (2003) Value of low dose combination treatment with blood pressure lowering drugs: Analysis of 354 randomised trials. British Medical Journal, 326, 1427-1431. doi:10.1136/bmj.326.7404.1427

[25] Degli Esposti, L., Degli Esposti, E., Valpiani, G., et al. (2002) A retrospective, population-based analysis of persistence with antihypertensive rug therapy in primary care practice in Italy. Clinical Therapeutics, 24, 1347-1357. doi:10.1016/S0149-2918(02)80039-X

[26] Degli Esposti, L., Di Martino, M., Saragoni, S., et al. (2004) Pharmacoeconomics of antihypertensive drug treatment: An analysis of how long patients remain on various antihypertensive therapies. The Journal of Clinical Hypertension, 6, 76-84. doi:10.1080/028418501127346846

[27] $\mathrm{Hu}, \mathrm{T}$. and Desai, J.P. (2004) Soft-tissue material properties under large deformation: Strain rate effect. Proceedings of the 26th Annual International Conference of the IEEE EMBS, San Francisco, 1-5 September 2004, 27582761.

[28] Ortega, R., Loria, A. and Kelly, R. (1995) A semiglobally stable output feedback PI2D regulator for robot manipulators. IEEE Transactions on Automatic Control, 40, 1432-1436. doi:10.1109/9.402235 
[29] Wit, E. and McClure, J. (2004) Statistics for microarrays: Design, analysis, and inference. 5th Edition, John Wiley \& Sons Ltd., Chichester.

[30] Prasad, A.S. (1982) Clinical and biochemical spectrum of zinc deficiency in human subjects. In: Prasad, A.S., Ed., Clinical, Biochemical and Nutritional Aspects of Trace Elements, Alan R. Liss, Inc., New York, 5-15.

[31] Giambastiani, B.M.S. (2007) Evoluzione idrologica ed idrogeologica della pineta di San Vitale (Ravenna). Ph.D.
Thesis, Bologna University, Bologna.

[32] Wu, J.K. (1994) Two problems of computer mechanics program system. Proceedings of Finite Element Analysis and $C A D$, Peking University Press, Beijing, 9-15.

[33] Honeycutt, L. (1998) Communication and design course. http://dcr.rpi.edu/commdesign/class1.html

[34] Wright, O. and Wright, W. (1906) Flying-machine. US Patent No. 821393. 